\title{
Research Paper: The Effect of 12 Weeks of Training in Water, on Land and Combined Environment on Bone Mineral Density in Premenopausal Women
}

\author{
Safoura Ghasemi ${ }^{*}$, Heydar Sadeghi ${ }^{1}$, Ahmad Tahamoli Roudsari $^{2}$, Zahra Basiri $^{2}$ \\ 1. Department of Sports Biomechanics, Faculty of Physical Education and Sport Sciences, Kharazmi University, Tehran, Iran \\ 2. Department of Rheumatology, School of Medicine, Hamadan University of Medical Sciences, Hamadan, Iran.
}

Citation: Ghasemi S, Sadeghi H, Tahamoli Roudsari A, Basiri Z. The Effect of 12 Weeks of Training in Water, on Land and Combined Environment on Bone Mineral Density in Premenopausal Women. Physical Treatments. 2016; 6(1):19-28. https://doi.org/10.18869/NRIP.PTJ.6.1.19

https://doi.org/10.18869/NRIP.PTJ.6.1.19

Article info:

Received: 13 Nov. 2015

Accepted: 28 Jan. 2016

Keywords:

Bone density, Water exercise, Land exercise, Combined environment exercise, Premenopause

\section{A B S T RA C T}

Purpose: Given that physical activity is the most important environmental moderating factor, it has been known as an effective, available, low-cost and non-pharmacological approach to increase or maintain bone density at different ages. The purpose of this study was to investigate the effect of 12 weeks of training in water, on land and combined environment on bone mineral density in premenopausal women.

Methods: In this quasi-experimental study with a pretest-posttest design and a control group, 40 premenopausal women aged between 40 and 45 years were divided into four groups (with 10 patients each) based on the exercise environment: water, land, combined, and controlled. Each group exercised three days a week for 12 weeks, with each session lasting 70 minutes. The lumbar vertebrae bone mineral density of the participants was measured by DEXA before and after 12 weeks and the data were analyzed using descriptive statistics, ANOVA, Analysis of covariance and LSD, with the significance level of .For statistical calculations, SPSS software version 21 was used.

Results: In the combined environment group, the lumbar vertebrae bone mineral density showed a significant increase $(\mathrm{P}>0.05)$, while in the control group first vertebra bone density loss was observed for the second and fourth lumbar vertebrae $(\mathrm{P}<0.05)$. In comparison with the control group, there was a significant difference among the first and fourth lumbar vertebrae bone mineral density in the water exercise group;first, third and fourth lumbar vertebrae density in the land exercise group; and all lumbar vertebrae density in the combined environment group.

Conclusion: Given that there was no difference observed between water and land exercise groups,exercise in any environment is recommended for premenopausal women because the least effect that exercise can have is prevention of bone loss in these ages.

* Corresponding Author: 


\section{Introduction}

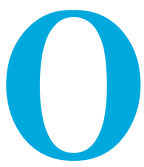

steoporosis is the most common metabolic bone disease that reduces bone mass density (BMD) and destroys its microstructure, which increases the risk of fractures. With aging, women lose $30-50 \%$ of trabecular bone mass and $25-30 \%$ of cortical bone mass. Women lose most of their bone mass during premenopause and postmenopause. The prevalence of osteopenia and osteoporosis among premenopausal women is $15 \%$ and $6 \%$, respectively [1] and the reported decrease in BMD for these women is between $0.25 \%$ and $1 \%$ per annum [2]. Studies have shown that maintaining optimal level of bone mineral density during premenopause is important for reducing the risk of osteoporosis and fractures during the post-menopause period, which is associated with an increased relative risk by 1.5 to 3 times [1].

There are different strategies for different age groups to increase or maintain bone density [2]. However, one effective, low-cost and non-pharmacological approach to achieve this goal is physical activity, which is the most important environmental factor modulator to increase or maintain bone mineral density in adults and children [3]. Habib-Zadeh et al. (2010) investigated the effect of walking on BMD (hip and lumbar vertebrae) of 40 obese and thin non-athlete girls and did not observe a significant effect on hip and waist BMD of the subjects [4].

Molhim (2004) showed that three months of jogging led to an increase in hip and spinal bone density and weight loss in obese, active women aged between 25 and 50 [5]. Kahn's study (2001) showed that bone mineral density is higher in organs that are tolerant of body weight [6]. Bassey and Ramsdale (1995) investigated the effect of weight-bearing exercise of high and low intensity within a year on postmenopausal women and did not observe a significant difference in BMD values of the subjects [7]. Kelley et al. (2001) showed the positive effect of stretching exercises on bone mineral density of the hip, radius and lumbar vertebrae of postmenopausal women [8].

Smidt et al. (1992) investigated the effect of a year of stretching exercises on postmenopausal women and did not observe a significant relationship between exercise and bone mineral density of the hip and lumbar vertebrae [9]. Chien et al. (2000) studied the effect of 24 weeks of aerobic exercises on bone density in postmenopausal women and showed a positive impact in increasing bone density in cervical, lumbar and thigh vertebrae [10]. Mousavian et al. (2015) examined the effect of Pilates exercise on osteoporosis of women aged 50 to 60 years and observed that 12 weeks of Pilates exercise increases the bone density of the hip and waist [11].

Most research has been carried out in a land environment, while exercise in water has a positive impact on coordination and perceptual, vestibular and visual systems [12]. Besides, a water environment imposes a resistance tailored to the needs of each individual's body, causes muscle activity and engages the larger muscle groups to overcome resistance. It also increases mechanical stress on bones and thus stimulates bone formation [13]. In this context, there are few studies on the impact of exercises in water on BMD. Vanaki et al. (2014) investigated the effect of 12 weeks of weight-bearing exercises in water on bone density of sedentary middle-aged women and concluded that exercise in water increased bone density [14]

Tofighi and Hefz Allesan (2010) examined the effect of 12 weeks of aerobic and resistance exercises in water on bone mineral density of postmenopausal obese women and observed a significant increase in bone mineral density [15]. Mohammadi (2013) reported in his master's thesis that 8 weeks of exercise on land and water increased bone density in postmenopausal women of 50-75 years of age [16]. However, Bravo et al. (1997) concluded that 12 weeks of weight-bearing exercises in water had no effect on bone mineral density of the thigh and caused a decrease in lumbar bone mineral density in postmenopausal women [17].

A review of the literature suggests that most studies have been conducted in a land or water environment and they show contradictory results. On the other hand, most researches have been carried out on postmenopausal women while a more appropriate approach would be to investigate the effect of exercise on bone density before menopause. Such a study could help prevent osteoporosis by designing optimum exercise strategies, including the type of exercise (endurance and strength, weight-bearing) and the environment (land, water or a combination of both) that is more suitable for a particular part of the body. Therefore, the aim of the present study was to investigate the effect of 12 weeks of exercise in water, on land and in a combined environment on bone mineral density in premenopausal women.

\section{Materials and Methods}

\section{Subjects}

The population of this quasi-experimental study pretest-posttest with the control group that is of applied type study consisted of premenopausal women of 40 to 45 years of age living in Hamadan in 2015. The research 
protocol was approved by the Research Ethics Committee and informed consent was obtained from the individuals. A questionnaire was distributed among 100 women. It included fields for age, height, weight, history of fractures later in life, medicine consumption, calcium intake, physical activity, number of deliveries, age of menarche, pregnancy and disease.

Based on a doctor's reference, 40 women with the inclusion criteria voluntarily participated in the study. The inclusion criteria consisted of the following: Lack of rheumatoid arthritis diseases, hypothyroidism or hyperthyroidism, parathyroid and adrenal disorders, diabetes mellitus, renal failure, advanced liver failure, cardiovascular disease, neurological disorders, traumatic brain injury, lower extremity injuries, menopausal symptoms, fractures, any type of cancer, menstrual disorders beginning after 18 years of age, permanent cessation of menstruation or cessation during the last quarter, less than 6 months period in the previous year, ovary removal before menopause and infertility or pregnancy or breast-feeding during the study period, smoking and alcohol consumption, drug addiction, deformity of the vertebrae, hospitalization due to illness during the two weeks prior to the study, complete bed rest for 3 consecutive months, consumption of estrogen and progesterone medicines, t-score less than -2.5 , and consumption of calcium, multivitamins and vitamin $\mathrm{D}$ pills and injections of vitamin $\mathrm{D}_{3}$.

The participants were divided in four groups of 10 people each based on the environment in which they were to exercise: in water, on land, in a combined environment, and as a control group. Each of the first three groups performed sport activities for 12 weeks, three times a week with each session lasting for 70 minutes. At least one day of rest was provided between sessions. The control group had no sport activities during these 12 weeks. All participants were asked to avoid food supplements, vitamin D tablets and calcium intake during the period of investigation and were prohibited from using any medicinal drugs (particularly those that affect bone density) without consulting their doctor.

\section{Exercise protocols}

\section{Exercise protocol in the water}

The water temperature was set between 29 and $30^{\circ} \mathrm{C}$ and the water level varied from the seventh cervical vertebra (first four weeks), to Xiphoid (fourth-eighth week) and the anterior superior iliac vertebrae to (ninth-twelfth week) [18]. When participants entered the water, they performed warming-up and stretching exercises (20 minutes), for the muscles of the lumbar region and the lower extremities. Resistance exercises (20 minutes) were performed without aid in the first four weeks with waterproof equipment being used in weeks 5 to 12 .

Bars, dumbbells and barbell were used to exercise the upper body resistance. These exercises included bench press, rotation of the waist, lumbar flexion and extension. On the other hand, foam pads were used to exercise the lower body resistance through plantar and ankle dorsiflexion, leg press, knee flexion and extension, hip abduction and adduction and hip flexion-extension. These aid tools cause resistance when the water moves. Since water resistance increases with faster movements, the participants performed the exercises in a range of motion sand at greater speed. To control the exercise intensity, the heart rate was measured at each session. Endurance exercises (20 minutes) in water included walking, hopping and jumping in different directions. Cool-down exercises in the water included muscle relaxation exercises such as floating and stretching [19, 20].

\section{Exercise protocol on land}

A 10-minute warm-up routine included walking, running at slow speed and stretching exercises. Strength exercises (35 minutes), with $50 \%$ in open kinetic chain and 50\% in closed kinetic chain, included 8 motions (stretching Latissimus, flexion of the knee, standing leg press, trunk extension, seated knee press, knee extension, seated leg press and sit-ups) [21-22]. For the first four weeks, with 1-2 sets of ten repetitions with an intensity of $60-65 \%, 1 \mathrm{RM}$ was considered. For the second four weeks with 3 sets of 8 repetitions and the intensity of 70-75\%, 1RM and for the third four weeks with 3 sets with 10 repetition sand intensity of $75-80 \% 1 \mathrm{RM}$ was considered for exercise [21-22]. A minute's rest was considered between sets. In addition to identifying $1 \mathrm{RM}$ before the exercise, the first of each month $1 \mathrm{RM}$ was repeated. Endurance exercises (15 minutes) included walking on the treadmill with $60-65 \%$ of maximum heart rate (30 seconds between each set) and cool down (10 minutes) including relaxation and stretching.

\section{Combined environment protocol}

It included 6 weeks of training in water and 6 weeks of training on land. Instead of dividing the exercise routine into three blocks of four weeks each, this type of exercise divided it into three blocks of two weeks each. Exercises during each two-week block were carried out based on the protocols established for water (first week) and land (second week).

\section{Tools and data collection}

To measure the height $(\mathrm{cm})$, a standing stadiometer with $1 \mathrm{~mm}$ accuracy (after a normal exhale) was used, 


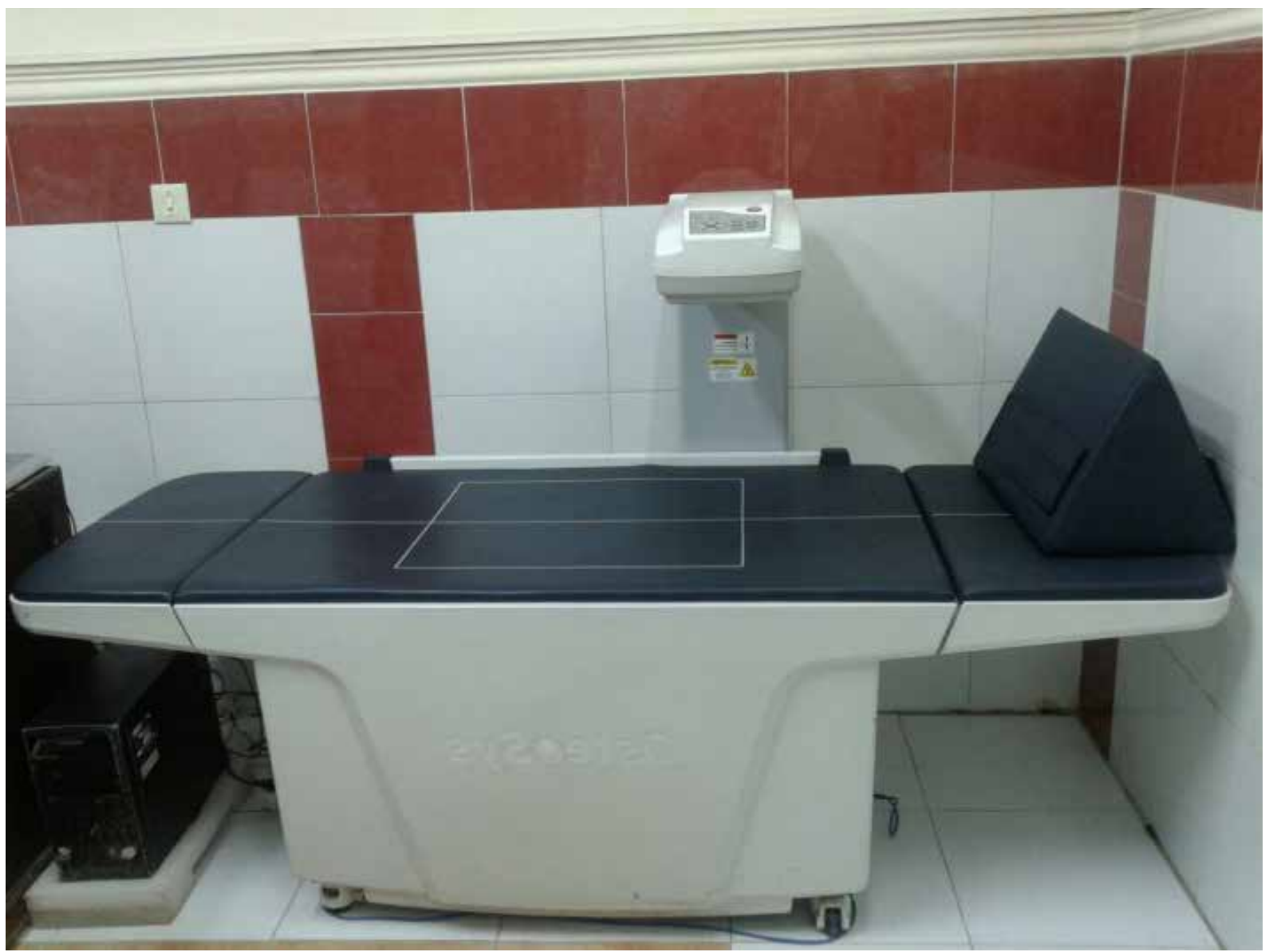

PHYSICAL TREA T MENTS

Figure 1. DEXA, X-ray Dexa, Model Dexxum-T, OSTEOSYS Co. made in South Korea.

while the weight $(\mathrm{kg})$ was recorded by using a digital scale with $0.01 \mathrm{~kg}$ accuracy with minimal clothing and without shoes. Bone density at two locations (Proximal femoral and lumbar vertebrae L1 to L4) were measured by DEXA with Dexa X-ray, model Dexxum-T of OSTEOSYS Co. made in South Korea (Figure 1), evaluated by a specialist. After obtaining the weight, bone densitometry was measured by the central apparatus, during which the subject lay on her back on the machine bed with the receiver of the device placed on the area of the body that was intended to measure bone density.

The X-ray was directed towards the bones of the lumbar vertebrae and the hip in the pelvic area. This method is simple, quick, non-invasive and painless; it does not require general or local anesthesia for testing and measures bone density within 10 to 20 minutes. Prior to the study, densitometry and its possible harmful side effects were explainedto all participants. Bone mineral density was calculated in grams per square centimeter and its results were instantly ready via computers connected to the device.
All tests related to bone density were investigated before and after the exercise period of 12 weeks, with a 48- to 72-hour interval after the last exercise session. For statistical analysis, the mean and standard deviation was used to describe the information, the KolmogorovSmirnov test was used for examining normality assumption for the measured parameters, and parametric ANOVA, analysis of covariance and LSD were used to investigate significant group differences. To perform the analysis of covariance, first analysis assumptions were examined. To ensure that the data for this study are the underlying assumptions to estimate covariance analysis, four assumptions of Covariance including the natural distribution of scores ( $\mathrm{P} \geq 0.05)$, Homogeneity of variances ( $\mathrm{P} \geq 0.05$ ), Conformance slope of regression ( $\mathrm{P} \geq 0.05$ ), and Pre-test and linear correlation and the dependent variable $(\mathrm{P} \leq 0.05)$ were reviewed and approved and the LSD post hoc test was used to compare the groups. Tests were analyzed at a significance level of 0.05 using SPSS version 21 . 


\section{Results}

As may be observed in the table related to the mean, standard deviation and anthropometric characteristics of the subjects (Table 1), ANOVA revealed that the participating groups were homogenous in terms of age, height, weight, and body mass index (BMI).

The mean and standard deviation of Bone Density of Lumbar Vertebrae L1, L2, L3 and L4 in the pre-test and post-test of water exercise, land exercise, combined exercise and control groups are provided in Table 2. Mean bone density increased in all the variables in water exercise, land exercise and combination groups, while in the control group bone density decreased in all the variables after 12 weeks.

The results of analysis of covariance (Table 2) for the first and fourth lumbar vertebrae variables (Lumbar Vertebrae), the first lumbar vertebrae (L1), the second lumbar vertebrae (L2), the third lumbar vertebra (L3) and fourth lumbar vertebra (L4) after the exercise shows that by removing the effect of pre-test and posttest scores, values of these variables are statistically different $(\mathrm{P}<0.05)$. Except for the first and fourth lumbar vertebrae (Lumbar Vertebrae), no significant difference was observed in the results of analysis of covariance in this variable among the groups $(\mathrm{P}<0.05)$.

Comparing the groups two-by-two (LSD) in Table 3 shows that in the Lumbar Vertebrae, only the combination group compared with the control group $(\mathrm{P}=0.19)$ showed a significant difference; in the other groups, no difference was observed in this variable. In the region of $\mathrm{L} 1$, water exercise group $(\mathrm{P}=0.008)$, land exercise $(\mathrm{P}=0.017)$ and combination $(\mathrm{P}=0.037)$ showed a significant difference compared with the control group and in comparing the two other groups, no significant difference was observed in this variable. In the L2 area, water exercise group $(\mathrm{P}=0.016)$ and combination group $(\mathrm{P}=0.002)$ showed a significant difference compared with the control group and in comparison of pairs of other groups no significant difference was observed in this variable. In the L3 area, water exercise group $(\mathrm{P}=0.013)$ and combination group $(\mathrm{P}=0.001)$ showed a significant difference compared with

Table 1. Anthropometric characteristics of the subjects participating in this study.

\begin{tabular}{|c|c|c|c|c|c|c|c|c|c|}
\hline & \multicolumn{2}{|c|}{ Group of Water Exercise } & \multicolumn{2}{|c|}{ Group of Land Exercise } & \multicolumn{2}{|c|}{ Combined Environment Group } & \multicolumn{2}{|c|}{ Control Group } & \multirow{2}{*}{$\mathbf{P}$} \\
\hline & SD & Mean & SD & Mean & SD & Mean & SD & Mean & \\
\hline Age & 1.64 & 43.30 & 2.20 & 42.20 & 1.72 & 42.50 & 1.84 & 43.50 & 0.35 \\
\hline Height & 3.62 & 158.11 & 7.87 & 158.10 & 6.52 & 160.72 & 6.46 & 160.53 & 0.66 \\
\hline Weight & 11.09 & 75.53 & 6.83 & 72.68 & 9.13 & 71.02 & 11.60 & 71.07 & 0.51 \\
\hline Body Mass Index* & 4.07 & 30.17 & 3.74 & 29.24 & 3.44 & 27.74 & 4.73 & 27.68 & 0.34 \\
\hline
\end{tabular}

*BMI: Proportion of weight to square of height $\left(\mathrm{Kg} / \mathrm{m}^{2}\right)$.

Table 2. Descriptive and analytical results of analysis of covariance in four groups and five bone density areas before and after exercise.

\begin{tabular}{|c|c|c|c|c|c|}
\hline Area & Test & Water & Land & Combination of Both & Control \\
\hline \multirow{2}{*}{$\begin{array}{c}\text { First to fourth lumbar } \\
\text { vertebrae }\end{array}$} & Post-test & $1.32 \pm 40.104$ & $1.263 \pm 0.102$ & $1.322 \pm 0.092$ & $1.253 \pm 0.154$ \\
\hline & Pre-test & $1.278 \pm 0.116$ & $1.226 \pm 0.093$ & $1.240 \pm 0.156$ & $1.279 \pm 0.200$ \\
\hline \multirow{2}{*}{$\begin{array}{l}\text { First lumbar vertebrae } \\
\text { (L1) }\end{array}$} & Post-test & $1.308 \pm 0.123$ & $1.251 \pm 0.079$ & $1.257 \pm 0.136$ & $1.223 \pm 0.131$ \\
\hline & Pre-test & $1.274 \pm 0.114$ & $1.204 \pm 0.117$ & $1.233 \pm 0.129$ & $1.294 \pm 0.145$ \\
\hline \multirow{2}{*}{$\begin{array}{c}\text { Second lumbar verte- } \\
\text { brae (L2) }\end{array}$} & Post-test & $1.333 \pm 0.124$ & $1.240 \pm 0.096$ & $1.327 \pm 0.163$ & $1.255 \pm 0.128$ \\
\hline & Pre-test & $1.291 \pm 0.094$ & $1.230 \pm 0.089$ & $1.251 \pm 0.139$ & $1.304 \pm 0.161$ \\
\hline \multirow{2}{*}{$\begin{array}{l}\text { Third lumbar verte- } \\
\text { brae (L3) }\end{array}$} & Post-test & $1.334 \pm 0.096$ & $1.210 \pm 0.108$ & $1.318 \pm 0.147$ & $1.235 \pm 0.164$ \\
\hline & Pre-test & $1.309 \pm 0.121$ & $1.201 \pm 0.118$ & $1.249 \pm 0.175$ & $1.274 \pm 0.183$ \\
\hline \multirow{2}{*}{$\begin{array}{c}\text { Fourth lumbar verte- } \\
\text { brae (L4) }\end{array}$} & Post-test & $1.309 \pm 0.121$ & $1.189 \pm 0.120$ & $1.264 \pm 0.221$ & $1.220 \pm 0.187$ \\
\hline & Pre-test & $1.301 \pm 0.107$ & $1.164 \pm 0.129$ & $1.215 \pm 0.239$ & $1.296 \pm 0.230$ \\
\hline
\end{tabular}




\begin{tabular}{|c|c|c|c|c|c|}
\hline Area & Resource & Sum of Square Type III & Degree of Freedom & F Statistics & P-Value \\
\hline \multirow{3}{*}{$\begin{array}{l}\text { First to fourth lumbar } \\
\text { vertebrae }\end{array}$} & Constant & 0.170 & 1 & 24.720 & 0.000 \\
\hline & Pre-test & 0.242 & 1 & 35.120 & 0.000 \\
\hline & Group & 0.047 & 3 & 2.290 & 0.095 \\
\hline \multirow{3}{*}{$\begin{array}{l}\text { First lumbar vertebrae } \\
\text { (L1) }\end{array}$} & Constant & 0.055 & 1 & 9.076 & 0.005 \\
\hline & Pre-test & 0.297 & 1 & 48.640 & 0.000 \\
\hline & Group & 0.060 & 3 & 3.272 & $0.032^{*}$ \\
\hline \multirow{3}{*}{$\begin{array}{l}\text { Second lumbar verte- } \\
\text { brae (L2) }\end{array}$} & Constant & 0.017 & 1 & 2.838 & 0.101 \\
\hline & Pre-test & 0.393 & 1 & 64.247 & 0.000 \\
\hline & Group & 0.076 & 3 & 4.156 & $0.013^{*}$ \\
\hline \multirow{3}{*}{$\begin{array}{l}\text { Third lumbar verte- } \\
\text { brae (L3) }\end{array}$} & Constant & 0.045 & 1 & 12.295 & 0.001 \\
\hline & Pre-test & 0.496 & 1 & 136.387 & 0.000 \\
\hline & Group & 0.059 & 3 & 5.447 & $0.002^{*}$ \\
\hline \multirow{3}{*}{$\begin{array}{l}\text { Fourth lumbar verte- } \\
\text { brae (L4) }\end{array}$} & Constant & 0.033 & 1 & 7.864 & 0.008 \\
\hline & Pre-test & 0.869 & 1 & 206.689 & 0.000 \\
\hline & Group & 0.068 & 3 & 5.389 & $0.004^{*}$ \\
\hline
\end{tabular}

*Significant difference in level of $\mathrm{P} \leq 0.05$.

PHYSICAL TREA TMENTS

the control group, the combined group had a significant difference with the land group $(\mathrm{P}=0.014)$. There was no significant difference in the other groups in this variable. In the area of $\mathrm{L} 4$, the water exercise group $(\mathrm{P}=0.06)$, the land exercise group $(\mathrm{P}=0.012)$, and the combination group $(\mathrm{P}=0.001)$ showed a significant difference compared with the control group.

\section{Discussion}

The current study has been conducted to investigate the effect of 12 weeks of training in water, on land and in a combined environment on bone mineral density of premenopausal women. Earlier studies have been conducted only on postmenopausal women. On the other hand, the current study took into consideration premenopausal women, and that too in specific training environments.

The study has been conducted on premenopausal women in three training environments (water, land and combination of both). The results showed that 12 weeks of exercise in all three environments increased bone mineral density of the lumbar vertebrae, though only the combined environment showed this increase to be significant (first to fourth lumbar vertebra and the second lumbar vertebrae).
In the control group, loss of bone mineral density of the lumbar vertebrae (first, second and fourth) was observed.

In comparison with the control group, there was a significant difference among the first and fourth lumbar vertebrae bone mineral density in the water exercise group; First, third and fourth lumbar vertebrae density in the land exercise group; And all lumbar vertebrae density in the combined environment group. While pair test results showed no statistical difference among the groups (excluding the difference observed between the combination training group with group of exercise on land in the second and third lumbar vertebrae).

The results of this study were consistent with the study by Kahn et al. (2001), which reported higher density in body weight bearing organs. In this study, it seems that exercise caused the increased density with some mechanical stress imposed on the lumbar vertebrae. Results of exercise on the land group showed significant increase in first, third and fourth vertebrae in comparison with the control group and there are similarities with the findings of Molhim (2004), who conducted the training for a similar period as in this study. However, the age of 
Table 3. The results of comparing groups two-by-two in LSD post hoc test.

\begin{tabular}{|c|c|c|c|c|c|}
\hline Areas of Bone Density & Groups & Groups & Mean Difference & SD & $\mathbf{P}$ \\
\hline \multirow{7}{*}{$\begin{array}{c}\text { First to fourth lumbar } \\
\text { vertebrae }\end{array}$} & & Water & -0.071 & 0.037 & 0.064 \\
\hline & Control & Land & -0.040 & 0.037 & 0.293 \\
\hline & & Combination & -0.091 & 0.037 & $0.019 *$ \\
\hline & & Land & -0.031 & 0.037 & 0.414 \\
\hline & Water & & & & \\
\hline & & Combination & -0.020 & 0.037 & 0.588 \\
\hline & Land & Combination & -0.051 & 0.037 & 0.176 \\
\hline \multirow{7}{*}{$\begin{array}{l}\text { First lumbar vertebrae } \\
\qquad \text { (L1) }\end{array}$} & & Water & -0.099 & 0.035 & $0.008 *$ \\
\hline & Control & Land & -0.091 & 0.036 & $0.017^{*}$ \\
\hline & & Combination & -0.077 & 0.035 & $0.037^{*}$ \\
\hline & & Land & 0.008 & 0.036 & 0.816 \\
\hline & Water & & & & \\
\hline & & Combination & 0.022 & 0.035 & 0.535 \\
\hline & Land & Combination & 0.014 & 0.035 & 0.699 \\
\hline \multirow{7}{*}{$\begin{array}{l}\text { Second lumbar verte- } \\
\text { brae (L2) }\end{array}$} & & Water & -0.088 & 0.035 & $0.016^{*}$ \\
\hline & Control & Land & -0.047 & 0.036 & 0.194 \\
\hline & & Combination & -0.116 & 0.035 & $0.002 *$ \\
\hline & & Land & 0.041 & 0.036 & 0.256 \\
\hline & Water & & & & \\
\hline & & Combination & -0.028 & 0.035 & 0.435 \\
\hline & Land & Combination & -0.069 & 0.035 & 0.057 \\
\hline \multirow{7}{*}{$\begin{array}{l}\text { Third lumbar verte- } \\
\text { brae (L3) }\end{array}$} & & Water & -0.071 & 0.027 & $0.013^{*}$ \\
\hline & Control & Land & -0.032 & 0.027 & 0.253 \\
\hline & & Combination & -0.102 & 0.027 & 0.001 \\
\hline & & Land & 0.039 & 0.027 & 0.169 \\
\hline & Water & & & & \\
\hline & & Combination & -0.031 & 0.027 & 0.267 \\
\hline & Land & Combination & -0.070 & 0.027 & $0.014 *$ \\
\hline \multirow{7}{*}{$\begin{array}{l}\text { Fourth lumbar verte- } \\
\text { brae (L4) }\end{array}$} & & Water & -0.085 & 0.029 & $0.006^{*}$ \\
\hline & Control & Land & -0.079 & 0.030 & $0.012 *$ \\
\hline & & Combination & -0.112 & 0.029 & $0.001 *$ \\
\hline & & Land & 0.006 & 0.030 & 0.840 \\
\hline & Water & & & & \\
\hline & & Combination & -0.027 & 0.029 & 0.372 \\
\hline & Land & Combination & -0.033 & 0.029 & 0.269 \\
\hline
\end{tabular}


participants in Molhim's study was between 25 and 50 years [5] and the study examined all the vertebrae [5].

Kelley et al. (2001) showed the positive impact of stretching exercises on the lumbar vertebrae [6] and the results were consistent with the findings of the present study. However, but the difference is that their sample comprised postmenopausal women and exercises were of stretching type, while the current study followed combined exercises, which had a significant positive effect on the entire vertebrae. The results of Chain (2000) were also consistent with the results of this study [10], but the duration of their study was twice that of the present study and their study was conducted on postmenopausal women. On the other hand, the present study showed positive results with a shorter duration and more pressure on younger women.

Results of this study are consistent with the study by Mousavian et al. (2015), which had a duration similar to the current study [11], but the exercises of their study were of Pilates type and performed on land by women of 60 to 65 years of age. In contrast, the exercises of this study were carried out in water, on land and in a combined environment on much younger subjects. In the present study, exercise on land had a positive effect only on the first, third and fourth lumbar vertebrae. In this study, results of the water exercise group showed a significant increase in L1 to L4 area compared with the control group, which is consistent with the results of a study by Vanaki et al. (2014), which was conducted over 12 weeks and in the form of weight bearing exercises in water [14].

However, the subjects in the research by Vanaki et al. were sedentary women aged 50 to 70 years and the study observed a significant effect on the lumbar vertebrae. In comparison, the subjects in this study were younger and the exercises just had a positive effect on single lumbar vertebrae and no significant effect on the first to fourth vertebrae. Tawfighi and Hefz Allesan (2010) investigated the effect of 12 weeks of aerobic and resistance exercises in water on the lumbar and hip vertebrae and observed a significant increase in hip bone mineral density [15]. Like the current research, they observed no significant difference in the entire level of the lumbar vertebrae. Their research was on obese postmenopausal women, a significant departure from this study.

The results of the current research can also be compared with the study by Mohammadi et al. (2013) on osteoporosis in postmenopausal women with an exercise regimen of 8 weeks in land and water environments [17]. In their study, exercise in water was more influential than exercise on land, while in the present study there is no statistical difference between exercise on land and water. In this study, in conjunction with the group of exercises in the combination environment, a significant increase was observed in all variables compared with the control group, but other similar studies are lacking.

Results of this research were inconsistent with a study by Habib-Zadeh et al. (2010) in which significant effects on bone mineral density was observed on obese and thin girls [4]. Possible causes of this inconsistency can be the shorter duration (two months), low-intensity workouts (walking with a heart rate of $50 \%$ to $75 \%$ ) or lower age and weight of subjects compared to the current study. The results of this research were inconsistent with Smidt et al. (1992) and Bassey and Ramsdale (1995) [7, 9]. Duration of exercises in their study was a year. It seems that the difference was probably due to the difference in intensity and age of participants (in their research postmenopausal women were investigated). Research by Bravo et al. (1997) was also inconsistent with our research. They observed that 12 weeks of weight-bearing exercises in water caused a decrease in lumbar bone mineral density in postmenopausal women [16]. Inconsistency of this research may be due to menopause and its negative effects on bone mineral density.

A holistic comparison of the current study with previous researches shows that there was consistency in some areas and exercise types and inconsistency in some variables. A reason for this could be that in previous researches the first and fourth lumbar vertebrae were considered generally and not evaluated separately. However, in this study, in addition to the first and fourth vertebrae, the individual vertebrae were investigated separately to provide more precise information on the effect of exercise on bone mineral density of the lumbar vertebrae.

According to the findings, although the exercise environment (water, land, or a combination of both) and study area (lumbar vertebrae in whole or discrete) are the significant factors affecting bone density, but in general, exercise has a positive effect on bone mineral density of the lumbar vertebrae. Therefore, the least effect that exercise can have is prevention of bone loss at an age approaching menopause.

Despite supplementation and drug control on the subjects of this study, the inability to control daily calorie intake in people over a period of 12 weeks was a limitation of this study. It is suggested that future researches need to consider additional information about the effect of exercise on muscles, bone density of the hip area at the same time with the lumbar vertebrae, and conduct the investigation with more number of subjects over a 
longer duration to investigate the follow-up effects of exercise on bone mineral density.

\section{Acknowledgments}

This paper was extracted from the first author' $\mathrm{PhD}$ dissertation, Department of Sports Biomechanics, Faculty of Physical Education and Sport Sciences, Kharazmi University, Tehran. The cooperation of all subjects participating in this study and officials of bone densitometry at Hamadan clinic, gym and pool is appreciated.

\section{Conflict of Interest}

The authors declared no conflict of interests.

\section{References}

[1] McDermott MT. Osteoporosis risk in premenopausal women. Pharmacotherapy. 2009; 29(3):305-17. doi: 10.1592/ phco.29.3.305

[2] Wolff I, van Croonenborg JJ, Kemper HC, Kostense PJ, Twisk JW. The effect of exercise training programs on bone mass: a meta-analysis of published controlled trials in pre- and postmenopausal women. Osteoporosis International. 1999; 9(1):1 12. doi: $10.1007 / \mathrm{s} 001980050109$

[3] Weeks BK, Young CM, Beck BR. Eight months of regular inschool jumping improves indices of bone strength in adolescent boys and girls: The power PE study. Journal of Bone \& Mineral Research. 2008; 23(7):1002-011. doi: 10.1359/jbmr. 080226

[4] Habibzadeh N. Preventing osteoporosis among underweight and obese sedentary young women. Baltic Journal of Health and Physical Activity. 2010; 2(2):97-103. doi: 10.2478/v101310009-6

[5] Mulhim AA, Gazzar SA, Bahnassy AA, Ali MS. Effect of exercise program on the bone mineral density in sedentary females. Bahrain Medical Bulletin. 2004; 26(2):1-5.

[6] Kun Z, Greenfield H, Xueqin D, Fraser DR. Improvement of bone health in childhood and adolescence. Nutrition Research Reviews. 2001; 14(1):119-52. doi: 10.1079/095442201108729169

[7] Bassey EJ, Ramsdale SJ. Weight-bearing exercise and ground reaction forces: A 12-month randomized controlled trial of effects on bone mineral density in healthy postmenopausal women. Bone. 1995; 16(4):469-76. doi: 10.1016/8756-3282 (95) 90193-0

[8] Kelley GA, Kelley KS, Tran ZV. Resistance training and bone mineral density in women: a meta-analysis of controlled trials. American Journal of Physical Medicine \& Rehabilitation. 2001; 80(1):65-77. doi: 10.1097/00002060-200101000-00017

[9] Smidt GL, Lin SY, O'dwyer KD, Blanpied PR. The effect of highintensity trunk exercise on bone mineral density of postmenopausal women. Spine. 1992; 17(3):280-5. doi: 10.1097/00007632199203000-00007
[10] Chien MY, Wu YT, Hsu AT, Yang RS, Lai JS. Efficacy of a 24week aerobic exercise program for osteopenic postmenopausa women. Calcified Tissue International. 2000; 67(6):443-8. doi: $10.1007 / \mathrm{s} 002230001180$

[11] Mousavian S, Sadeghi H, Taghavi M. [The effects of 12 sessions of Pilates exercise on bone loss in women (50 to 60 years) (Persian)]. Paper presented at: The $1^{\text {st }}$ National Conference on Advances in Physical Education and Sports; 2015 September 12-13; Chabahar, Iran.

[12] Devereux K, Robertson D, Briffa NK. Effects of a water-based program on women 65 years and over: a randomised controlled trial. Australian Journal of Physiotherapy. 2005; 51(2):102-08. doi: 10.1016/s0004-9514 (05) 70038-6

[13] Littrell TR, Snow CM. Bone density and physical function in postmenopausal women after a 12-month water exercise intervention. Medicine \& Science in Sports \& Exercise. 2004; 36:28990. doi: 10.1097/00005768-200405001-01388

[14] Vanaky B, Sadeghi H, Ramezani N. The effect of 12 weeks weight bearing water training on the bone density of middle age sedentary women. Biosciences Biotechnology Research Asia. 2014; 11(2):931-36. doi: 10.13005/bbra/1361

[15] Tofighi A, Hefz Alsan M. [The effect of a period of twelve weeks of aerobic and resistance exercise on bone mineral density in the lumbar vertebrae and femoral water and obese postmenopausal women (Persian)]. Olympic Quarterly; 2010 18(4):153-64

[16] Bravo G, Gauthier P, Roy PM, Payette H, Gaulin P. A weightbearing, water-based exercise program for osteopenic women Its impact on bone, functional fitness, and well-being. Archives of Physical Medicine and Rehabilitation. 1997; 78(12):1375-80. doi: 10.1016/s0003-9993 (97) 90313-0

[17] Mohamadi S. [Effect of 8 weeks training in water and land on osteoporosis of middle ages women 50 to 75 years old of Dezfool (Persian) ] [MSc. thesis]. Tehran: Islamic Azad University, Central Branch; 2013.

[18] Takeshima N, Rogers ME, Watanabe E, Brechue WF, Okada A, Yamada T, et al. Water-based exercise improves health-related aspects of fitness in older women. Medicine and Science in Sports \& Exercise. 2002; 34(3):544-51. doi: 10.1097/00005768200203000-00024

[19] Brennan D. Aquatic exercise in physical medicine \& rehabilitation. Oklahoma: Orthopedic Hospital Oklahoma; 2003.

[20] Elsisi HF, Mousa GS, Eldesoky MT. Electromagnetic field versus circuit weight training on bone mineral density in elderly women. Clinical Interventions in Aging. 2015; 10:539-47. doi: 10.2147/CIA. S78485

[21] Liang MT, Braun W, Bassin SL, Dutto D, Pontello A, Wong $\mathrm{ND}$, et al. Effect of high-impact aerobics and strength training on BMD in young women aged 20-35 years. International Journal of Sports Medicine. 2011; 32(2):100-08. doi: 10.1055/s-0030-1268503

[22] Kisner C, Colby LA. Therapeutic exercise: Foundations and techniques. Philadelphia: F. A. Davis Company; 2012. 
- 